\title{
Role of the Applied Magnetic Field on the Microstructural Evolution in Alnico 8 Alloys
}

\author{
LIN ZHOU, M.K. MILLER, H. DILLON, A. PALASYUK, S. CONSTANTINIDES, \\ R.W. MCCALLUM, I.E. ANDERSON, and M.J. KRAMER
}

\begin{abstract}
Improving performance of permanent magnet (PM) alloys is a complex optimization of microstructure through control of chemistry and processing. Alnico alloys represent a class of chemically complex alloys whose functionality as a PM is dependent on the nano-structuring which develops during thermal heat treatments in an applied magnetic field. Initial phase separation during rapid quenching of a solid solution from $1523 \mathrm{~K}\left(1250^{\circ} \mathrm{C}\right)$ results in a network structure consisting of interpenetrating continuous Fe-Co $\left(\alpha_{1}\right)$ and AlNi-based $\left(\alpha_{2}\right)$ phases. Annealing polycrystalline alnico with a fixed external magnetic field $(\mathbf{H})$ at $1073 \mathrm{~K}\left(800{ }^{\circ} \mathrm{C}\right)$ causes isolation, coarsening, and faceting of the $\alpha_{1}$ phase with increasing time. Also, the volume fraction and the $\mathrm{Al}, \mathrm{Ni}, \mathrm{Ti}$, and $\mathrm{Cu}$ contents of the $\alpha_{1}$ phase decreased, while the $\mathrm{Fe}$ and $\mathrm{Co}$ contents increased with increasing field annealing time. A Cu-rich phase forms along the adjacent corners of two $\alpha_{1}$ facets in the $\alpha_{2}$ phase during the phase separation and coarsening process. The phase distribution and micro-magnetic domain structure is closely related to the relative orientation between $\mathbf{H}$ and the principal axes of each grain. The $\alpha_{1}$ rods are elongated in each grain along the $\langle 100\rangle$ direction with projected $\mathbf{H}$, where the preferentially extended length of the $\alpha_{1}$ rods along the selected $\langle 100\rangle$ is proportional to the magnitude of projected $\mathbf{H}$.
\end{abstract}

DOI: $10.1007 / \mathrm{s} 40553-013-0004-3$

(C) The Minerals, Metals \& Materials Society and ASM International 2014

\section{INTRODUCTION}

PERMANENT magnets (PM) with rare earth alloys, such as dysprosium and neodymium, are critical for PM-based motors and generators for hybrid electric vehicles and wind turbines. Recent concern for supply and price of the rare earth alloys has stimulated the search for alternative magnetic materials. ${ }^{[1]}$ Alnico, the first modern PM magnetic alloy, an alloy of primarily $\mathrm{Al}, \mathrm{Ni}, \mathrm{Co}$, and $\mathrm{Fe}$ with excellent magnetic stability at high temperature, is an attractive near-term non-rare earth PM alloy if modest increase in coercivity can be achieved. Alnico was discovered in $1932^{[2]}$ and optimized before 1970s mostly by empirical studies without the help of today's advanced characterization and simulation tools. ${ }^{[3-10]}$ The key to the optimization of magnetic properties is control of the aging process of the Fe-Co ferromagnetic $\left(\alpha_{1}\right)$ phase and a non-magnetic NiAl-based $\left(\alpha_{2}\right)$ phase. The highest energy product alnico alloys are 5-7 and 9, which are both grain aligned and heat-treated with an applied magnetic field. On the other hand, magnetic field-annealed alnico 8 without

LIN ZHOU, Assistant Scientist, H. DILLON, Graduate Student, A. PALASYUK, Research Scientist, R.W. MCCALLUM, Senior Materials Scientist, I.E. ANDERSON, Senior Metallurgist, and M.J. KRAMER, Senior Scientist, are with the Ames Lab, Ames, IA 50014. Contact e-mail: mjkramer@ameslab.gov M.K. MILLER, Corporate Fellow, is with the Oak Ridge National Laboratory, Oak Ridge, TN 37831-6139. S. CONSTANTINIDES, Director of Technology, is with the Arnold Magnetic Technologies Corporation, Rochester, NY 14625.

Manuscript submitted November 9, 2013.

Article published online January 24, 2014 aligned grains has the highest coercivity $\left(H_{\mathrm{ci}}\right)$. To further improve properties of alnico alloys, understanding and ultimately control of the nanostructure is key, especially the effect of applied magnetic field during heat treatment on morphology and magnetic domain structure. ${ }^{[2]}$ This study provides a detailed chemical and structural characterization of the $\alpha_{1}$ and $\alpha_{2}$ phases in alnico 8 alloy with different heat-treatment processes. Atom probe tomography (APT) is used for high-accuracy chemical determination and elemental mapping at very high spatial resolution in the structures. Comprehensive transmission electron microscopy (TEM) techniques, including diffraction contrast TEM and scanning transmission electron microscopy (STEM), provide details of the structure of the phases. Lorentz microscopy reveals the importance of the phase morphology and magnetic domain structure on controlling coercivity. Orientation imaging microscopy quantifies the relative orientation relationship between a single grain and applied external magnetic field during annealing. Since alnico 8, by class, has the highest $H_{\mathrm{ci}}$, this is the alloy of choice to try to understand the details of the phase evolution as a function of time and temperature.

\section{EXPERIMENTAL DETAILS}

The commercially optimized alnico 8 alloy (Fe30Co40.1-Ni-13-Al-7.1-Cu-3.0-Ti6.5, in wt pet) was supplied by Arnold Magnetic Technologies Corp. The alloy is polycrystalline with random grain orientation and had been thermo-magnetically aligned using their standard method. Our heat treatments of smaller samples cut 
from the larger single commercial sample was designed to evaluate the phase evolution of the critical processing steps: solutionizing and thermo-magnetic annealing. Samples with a size of $3 \mathrm{~mm}$ dia. $\times 8 \mathrm{~mm}$ in length were cut from the initial $20 \mathrm{~mm}$ x $100 \mathrm{~mm}$ cylinder. First, the smaller samples were solutionized at $1523 \mathrm{~K}$ $\left(1250^{\circ} \mathrm{C}\right)$ for 20 minutes in vacuum $\left(1.3 \times 10^{-4} \mathrm{~Pa}\right)$ then quenched into an oil bath (sample A, as solutionized). Samples were then annealed at $1073 \mathrm{~K}\left(800{ }^{\circ} \mathrm{C}\right)$ for 1.5 minutes (sample B) and 10 minutes (sample C), separately, with an external $1 \mathrm{~T}$ magnetic field.

The magnetic properties were measured using a Laboratorio Elettrofisico Engineering Walker LDJ Scientific AMH-5 Hysteresis graph with a $5 \mathrm{~mm}$ coil and a maximum applied field of $5.0 \mathrm{kOe}$ at room temperature in a closed-loop setup. Some metrics of interest are the saturation magnetization $\left(M_{\mathrm{s}}\right)$, the induction $(\mathbf{B})$, and the value of the applied field $(\mathbf{H})$ needed to reduce the magnetization to zero, i.e., coercivity $\left(H_{\mathrm{cB}}\right)$. The $M_{\mathrm{s}}$ is a measure of the total moment that the magnetic phase contributes and is a measure of the magnetic phase fraction that has formed. The intrinsic coercivity, $H_{\mathrm{ci}}$, is the ability of a magnet to maintain its magnetization in the presence of an opposing magnetic field, which is desirable to optimize without any significant reduction in $\mathbf{B}$.

Sample chemistry and microstructure were analyzed by TEM and APT. TEM analysis was performed on transverse sections (with electron beam parallel to $\mathbf{H}$ direction) for all the samples and on longitudinal orientation (with electron beam perpendicular to $\mathbf{H}$ direction) for the commercial sample. TEM samples were prepared by mechanical wedge-polishing followed by a short time, low-voltage Ar ion-milling with liquid nitrogen cold stage. The longitudinal TEM sample was intentionally cut and polished with its thin edge perpendicular to the $\mathbf{H}$ direction during heat treatment. Then the sample was transferred into a $\mathrm{Cu}$ washer, with $\mathbf{H}$ perpendicular to the long edge of the slot, as shown in Figure 1(a). Electron backscattered diffraction mapping also known as orientation imaging microscopy (OIM) mapping of the TEM thin edge was performed on Amray 1845 FESEM. A FEI Tecnai F20 (200 kV, FEG) with Lorentz lens was used for microstructural characterization. APT is an accurate and sensitive method for investigating details of the morphology and compositions of the phases present at near atomic scale. ${ }^{[11]}$ The APT was performed with a CAMECA Instruments Inc. LEAP $4000 \times$ HR on samples prepared with a FEI Nova 200 dual-beam focused ion beam (FIB) system. Samples were extracted from the interior of the grains from polished transverse sections by standard lift-out technique $^{[12]}$ so as to provide multiple interphase interfaces along the sample axis. The extents of the coexisting phases were revealed by isoconcentration surfaces.

\section{RESULTS}

Sample A (as solutionized) shows coarse $(\sim 170 \mu \mathrm{m})$ random grain structure as shown in both the OIM image (Figure 1(b)) and in the SEM using backscattered electron (BSE) images (Figure 2), similar to the
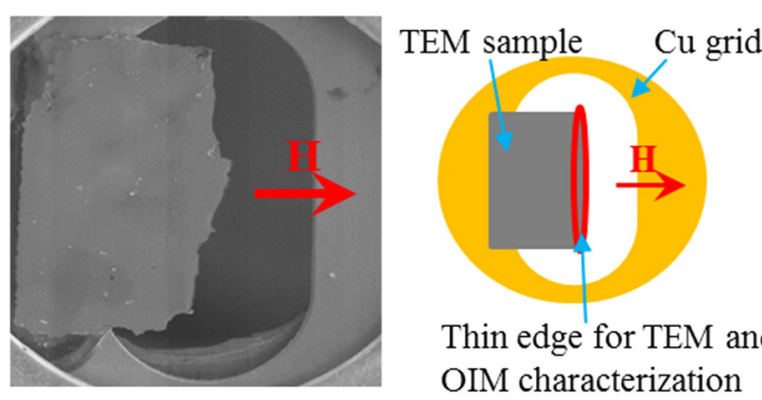

Thin edge for TEM and OIM characterization

(a)

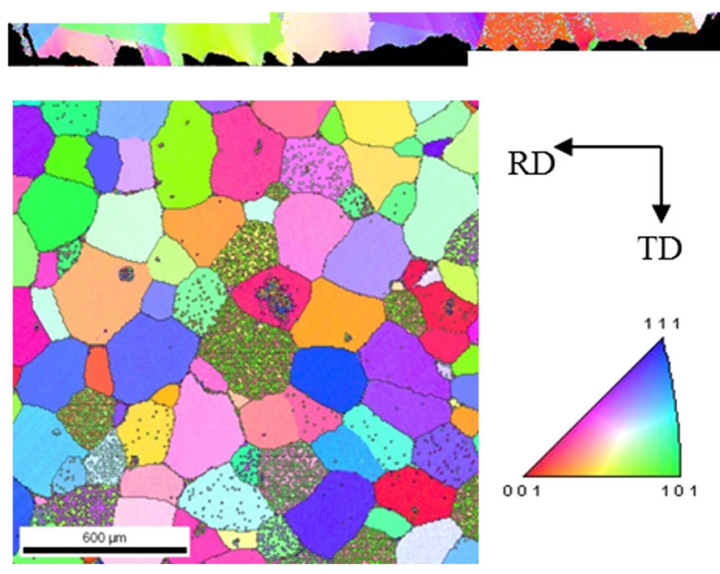

(b)

Fig. 1-(a) SEM image and the corresponding schematic showing relationship between $\mathbf{H}$ and TEM sample edge. (b) Inverse [001] pole figure of the commercial alnico 8 sample.

as-received commercial alloy. ${ }^{[13]}$ BSE images of sample A show that the grain size distribution is not uniform and some additional phases were present. For example, Ti-rich particles (dark dots in Figure 2(a)) were observed in the interior of some grains. The grain boundary region (denoted by the red dot in Figure 2(a)) is enlarged in Figure 2(b). This region exhibited both TiP (dark gray) and Fe-Co phases, consistent with the as-received commercial alnico 8 sample. While not common, these grain boundary phases are mostly likely from minor additions such as $\mathrm{S}$ or in some cases trace elements or impurities such as $\mathrm{P}$, in commercial ferrous alloys.

\section{A. Standard Production Commercial Alnico 8}

A bright-field TEM image, Figure 3(a), of a standard production commercial alnico 8 taken along transverse direction shows a nanometer scale "mosaic tile" structure with a characteristic size $\sim 40 \mathrm{~nm}$. The darker regions are the $\alpha_{1}$ phase, whereas the brighter regions are the $\alpha_{2}$ phase. The corresponding selected area electron diffraction pattern (SAED) (Figure 3(b)) indicates that the $\alpha_{1}$ phase is faceted mostly on $\{110\}$ and occasionally on $\{100\}$ planes. In general, when the $\alpha_{1}$ phase has $\{100\}$ planes facets, it will have a larger particle size. The $\alpha_{2}$ phase is an L2 ${ }_{1}$ ordered phase. ${ }^{[13]}$ HAADF STEM imaging, which emphasizes regions of high atomic number with brighter contrast because of greater electron scattering to large detector collection 


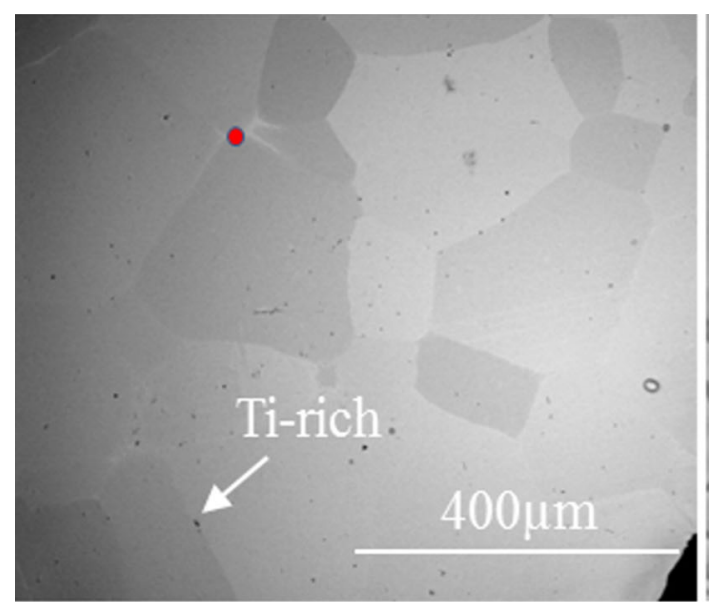

(a)

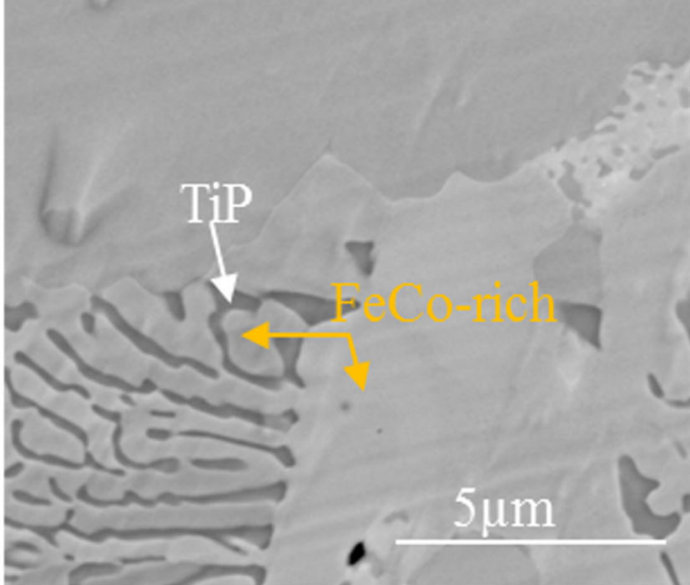

(b)

Fig. 2-SEM images of sample A (as-quenched). (a) Low mag image; (b) magnified image of the red dot region (Color figure online).

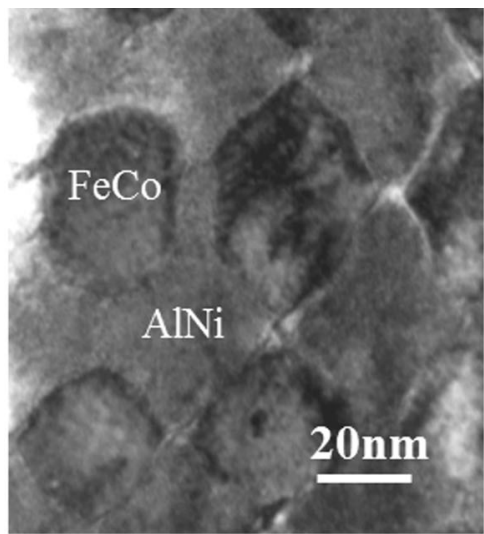

(a)

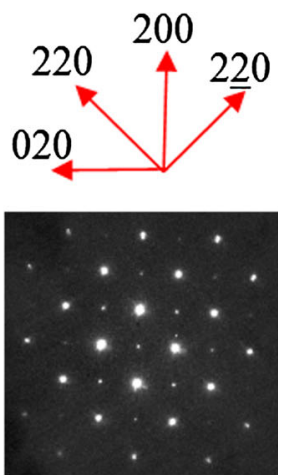

(b)

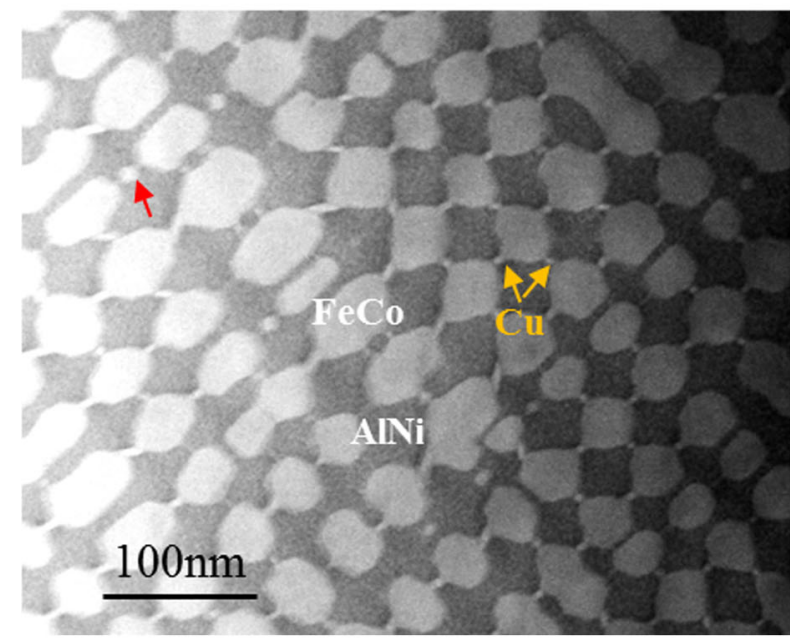

(c)

Fig. 3-(a) Bright-field TEM image of alnico 8 taken under [100] zone axis and $(b)$ the corresponding diffraction pattern. (c) HAADF STEM image of alnico 8 looking along transverse direction.

angles, ${ }^{[14]}$ clearly differentiates phase morphology in alnico alloys. Isolated cuboidal bright $\alpha_{1}$ phase embedded in continuous gray $\alpha_{2}$ phase is resolved, as shown in
Figure 3(c). $\alpha_{1}$ nodule $(\sim 10 \mathrm{~nm})$ between two big $\alpha_{1}$ patches was shown as well, as indicated by the red arrow in Figure 3(c). Small bright $\mathrm{Cu}$-rich particles $(\sim 2$ to $5 \mathrm{~nm}$ ) lie along the corners of two $\{110\}$ facet of the $\alpha_{1}$ phase.

The large thin region of the TEM specimen coupled with the random grain orientation of this sample (Figure 1) allows for a direct determination of the effect of applied magnetic field on morphology of the phases and micro-magnetic domain structure with different grain orientations. The TEM sample was intentionally loaded into the SEM for OIM analysis such that transverse direction (TD) is parallel to the applied field, rolling direction (RD) is along the edge of the foil, and the normal direction (ND) is the foil normal. An inverse [001] pole figure map of alnico 8 taken along the transverse direction is shown in Figure 1(b). Grain orientations can be seen from inserted color-coded stereographic triangle confirming the random grain orientation. Since the sample was prepared so that the applied field is normal to the edge of the thin TEM foil, approximately to within a few degrees, and in the plane of the foil and the foil normal is invariant (i.e., there was no serious sample bending), then the inverse pole figures represent a rigid body rotation of the principal bcc lattice to the OIM cardinal coordinates, as shown in Figure 4(a). There are three $\langle 100\rangle$ variants of the preferred growth of the decomposition for cubic structure. ${ }^{[15,16]}$ The magnitude of projected $\mathbf{H}$ to $\mathrm{a}\langle 100\rangle$ direction is proportional to $\sim \cos (\theta) \sin (\phi)$.

The mm-sized large thin area allowed the sampling of more than ten grains with different orientations, three representative grains labeled as I to III are presented in Figure 5. The corresponding HAADF STEM images, Lorentz TEM images, and the OIM [001] pole figures of those grains are shown in Figure 5. The $\cos (\theta) \sin (\phi)$ value was calculated for each $\langle 001\rangle$ directions and summarized in Table I. For grain I, $\cos (\theta) \sin (\phi)$ value along [100] direction (0.977) is much larger than that along [010] (0.167) and [001] (0.142) directions. The corresponding HAADF STEM imaging of grain I 


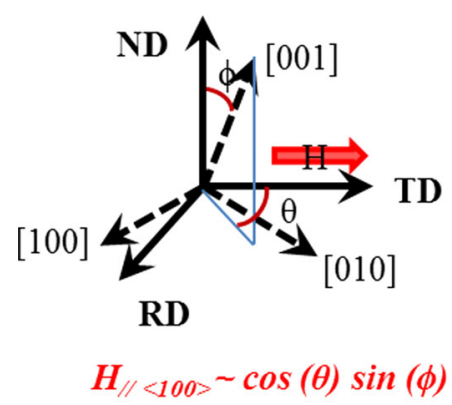

(a)

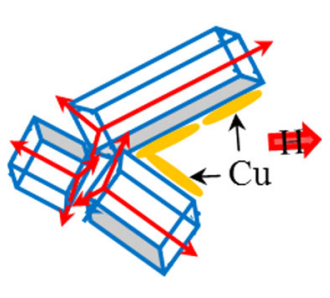

(b)

Fig. 4 - (a) Schematic showing orientation relationship between grain orientation, applied external magnetic field, and OIM axis. (b) Schematic showing distribution of $\mathrm{FeCo}$ rods in alnico 8 commercial alloy.

reveals that the $\alpha_{1}$ phase was elongated along [100] direction with length of $\sim 1 \mu \mathrm{m}$, while elongation along [010] and [001] directions was only occasionally observed with much shorter length $(<100 \mathrm{~nm})$. The corresponding Lorentz microscopy image, Figure 5(b), shows stripe shape micro-magnetic domains with length and width of $\sim 1 \mu \mathrm{m}$ and $\sim 100 \mathrm{~nm}$, respectively, parallel to the dominant $\alpha_{1}$ phase orientation. For grain II, the [100] and [010] directions have almost equal larger $\cos (\theta) \sin (\phi)$ value $(0.643$ and 0.759 , respectively) compared to the [001] direction. Elongated $\alpha_{1}$ cuboids with a length of $\sim 500 \mathrm{~nm}$ along [100] or [010] directions were observed, as shown in Figure 5(d). The corresponding Lorentz image shows block-shaped micro-magnetic domains corresponding to the patchwork pattern observed for the $\alpha_{1}$ phases. For grain III, all the three $\langle 100\rangle$ directions show close $\cos (\theta) \sin (\phi)$ values $(0.565$, 0.582 , and 0.601 , respectively). The corresponding HAADF STEM image indicates uniform elongation of $\alpha_{1}$ cuboids along all the three $\langle 100\rangle$ directions with a length $\sim 80 \mathrm{~nm}$. The Lorentz image of grain III shows a similar pattern in the micro-magnetic domains as seen in the $\alpha_{1}$ phases for this grain. The above results indicate that the $\alpha_{1}$ cuboids were not elongated along the applied magnetic field direction, as indicated by red arrow, but along the $\langle 100\rangle$ crystallographic directions with projected $\mathbf{H}$, as shown schematically in Figure 4(b). This has profound implications when an applied magnetic field is used to bias growth directions of the $\alpha_{1}$ phase. ${ }^{[17]}$

\section{B. Heat-Treated Commercial Alnico 8}

To better understand how the $\alpha_{1}$ and $\alpha_{2}$ phase coarsened after spinodal decomposition in the alnico 8 alloy, both the magnetic hysteresis loops and microstructure of this same alloy were investigated after different heat-treatment process as described above. The heat treatment at $1523 \mathrm{~K}\left(1250{ }^{\circ} \mathrm{C}\right)$ should be sufficient to erase the existing nanostructure. ${ }^{[2]}$

The interpenetrating nature of the $\alpha_{1}$ phase and the $\alpha_{2}$ phase is shown in APT results for the heat-treated alloys (Figure 6). In sample A, the $\alpha_{1}$ and the $\alpha_{2}$ phases are continuous with meandering boundary (except for a small number of regions at the edges of the volume) in the entire volume analyzed. The $\alpha_{1}$ phase starts to show some segmentation in sample $\mathrm{B}$, as shown in Figure 6(b). However, this segmentation may be due to the limited field of view. Isolated $\alpha_{1}$ blocks embedded in continuous $\alpha_{2}$ phase become clearly visible in sample C. $\mathrm{Cu}$ strongly partitions to the $\alpha_{2}$ phase, and $\mathrm{Cu}$-rich particles were observed from samples A to C. Assuming that the precipitated $\mathrm{Cu}$-rich particles are spherical particles, their average diameter slightly increased from $1.0 \mathrm{~nm}$ in sample A to $1.5 \mathrm{~nm}$ in sample C.

The isoconcentration surfaces may also be used to capture all the atoms within the three phases to estimate their average compositions from the total number of atoms collected from the phases. The results are summarized in Table II. The partitioning of $\mathrm{Fe}$ and Co to the $\alpha_{1}$ phase, and the $\mathrm{Al}, \mathrm{Ni}, \mathrm{Ti}$, and $\mathrm{Cu}$ to the $\alpha_{2}$ phase is clearly evident with increasing annealing time. From samples $\mathrm{A}$ to $\mathrm{C}$, the average $\mathrm{Cu}$ levels from the lassoed atoms from the isoconcentration surfaces in the $\mathrm{Cu}$-rich phase are similar $(\mathrm{Cu}: \sim 20.5 \mathrm{pct})$. However, the proximity histograms ${ }^{[18]}$ show a small increase in $\mathrm{Cu}$ at the core regions. The $\mathrm{Fe}$ and Co concentration increase, but the $\mathrm{Al}, \mathrm{Ni}, \mathrm{Ti}$, and $\mathrm{Cu}$ concentrations decrease with increasing annealing time in the $1 \mathrm{~T}$ field in the $\alpha_{1}$ phase. A rough estimation of volume ratio of $\alpha_{1}$ phase shows a decreasing trend from samples A to $\mathrm{C}$.

HAADF STEM imaging of samples A to $\mathrm{C}$ looking along transverse direction, as shown in Figure 7, confirmed that the $\alpha_{1}$ phase coarsened and faceted with annealing. The $\alpha_{1}$ phase in sample A (Figure 7(a) has a smaller dimension (diameter $\sim 8 \mathrm{~nm}$ ) with no obvious faceting and appears to be continuous phase as suggested by the APT. The $\alpha_{1}$ phase coarsens to a diameter of $\sim 12 \mathrm{~nm}$ after 90 seconds annealing at $1073 \mathrm{~K}$ $\left(800{ }^{\circ} \mathrm{C}\right)$ in sample B. Some of the $\alpha_{1}$ particles in sample $\mathrm{B}$ showed a tendency to faceting as they grow. The morphology of $\alpha_{1}$ phase in sample $C$ is similar to commercial alnico 8 with $\{110\}$ and $\{100\}$ faceting and $\mathrm{Cu}$-rich particles at the edge of two $\{110\}$ facets in the $\alpha_{2}$ phase. But the size of $\mathrm{Cu}$-rich particles in sample $\mathrm{C}$ is smaller than that in commercial alnico 8 . The $\alpha_{1}$ phase in sample $C$ has a diameter of $\sim 20 \mathrm{~nm}$, indicating coarsening of $\alpha_{1}$ phase occurs rapidly at $1073 \mathrm{~K}\left(800^{\circ} \mathrm{C}\right)$.

The size, shape, and connectivity of the $\alpha_{1}$ phase will all have a bearing on the coercivity while the phase fraction of the $\alpha_{1}$ phase and its $\mathrm{Fe}+\mathrm{Co}$ content will determine the $M_{\mathrm{s}}$. The orientation will determine $B_{\mathrm{r}}$. The measured hysteresis loops of samples A to $\mathrm{C}$, together with the commercial alnico 8 alloy are shown in Figure 8. The as-quenched alnico 8, i.e., sample A, has intrinsic coercivity $\left(H_{\mathrm{ci}}\right) \sim 60$ Oe indicating lack of any isolated $\alpha_{1}$ phases. The saturation magnetization $\left(M_{\mathrm{s}}\right)$ of $\sim 1.1 \mathrm{~T}$ is consistent with the higher fraction of $\alpha_{1}$ phase. For samples $\mathrm{B}$ and $\mathrm{C}$, the $H_{\mathrm{ci}}$ values increased significantly and reached $\sim 746$ and $\sim 788 \mathrm{Oe}$, respectively. The residual inductance values of the samples also notably improved in comparison to untreated sample A, i.e., $B_{\mathrm{r}}=\sim 0.74$ and $B_{\mathrm{r}}=\sim 0.87 \mathrm{~T}$, respectively. Although the saturation magnetizations $M_{\mathrm{s}}$ of all the samples exhibit rather similar values that comprise in rather narrow range of $B_{\mathrm{r}}=\sim 1.0$ to $\sim 1.1 \mathrm{~T}$, it is worth noticing that the sample $B$ which was heat-treated for 


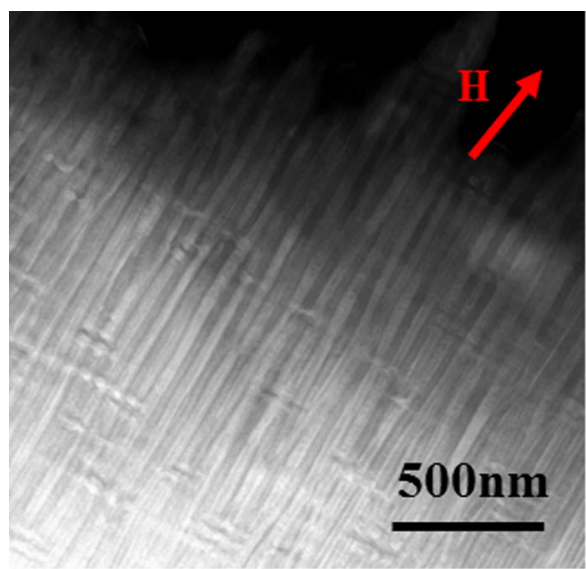

(a)

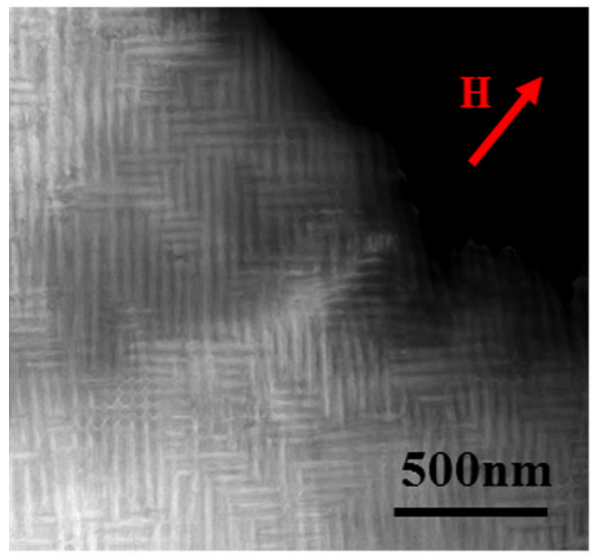

(d)

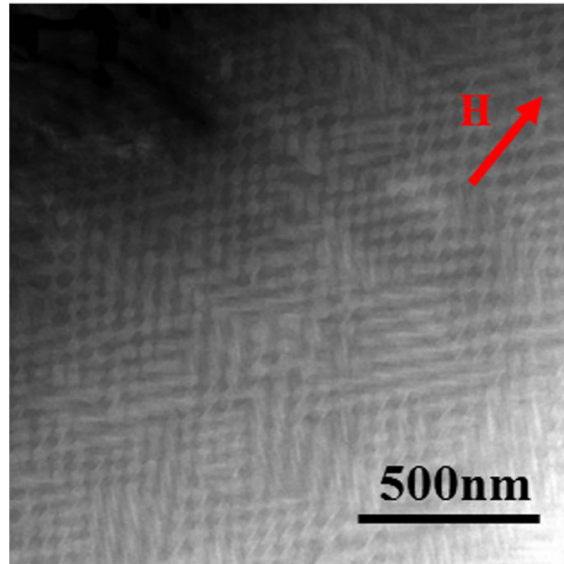

(g)

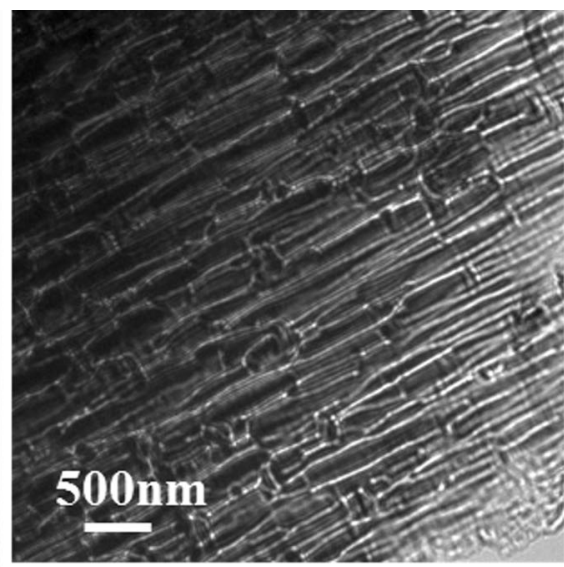

(b)

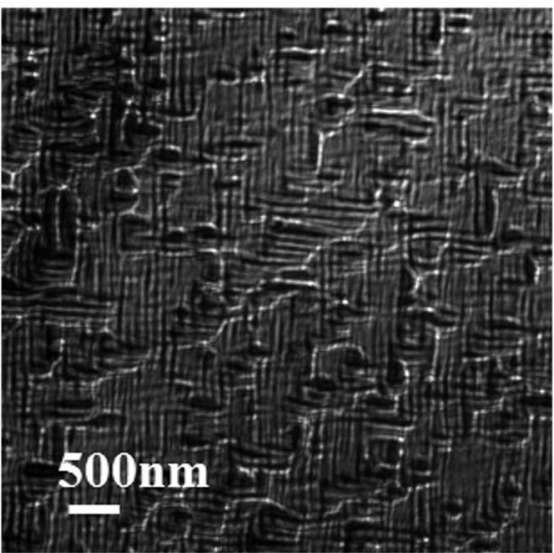

(e)

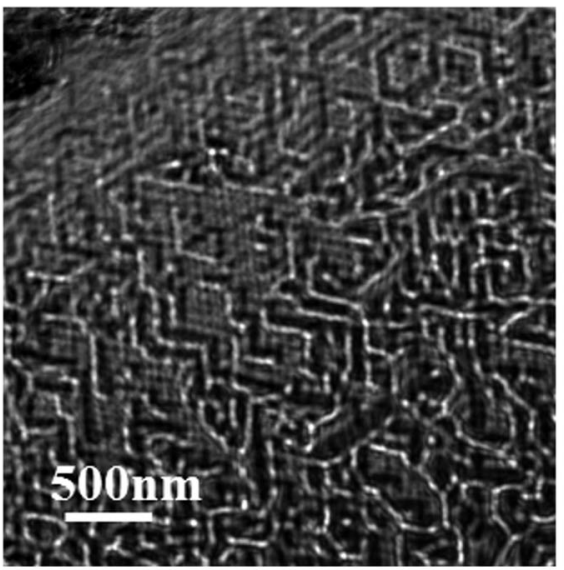

(h)

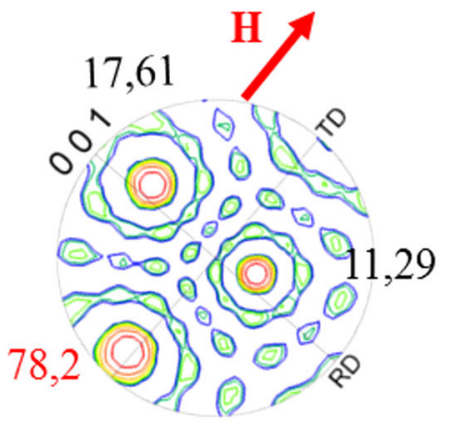

(c)

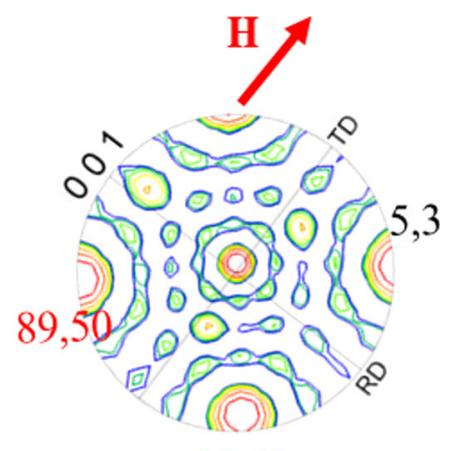

82,40

(f)

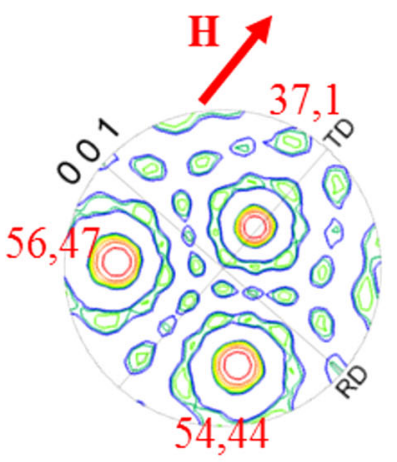

(i)

Fig. 5-HAADF STEM image of grain I $(a)$, II $(d)$, III $(g)$. Lorentz image of grain I $(b)$, II $(e)$, III $(h)$. The corresponding [001] pole figures of grain I $(c)$, II $(f)$ and III $(i)$. The applied magnetic field $(\mathbf{H})$ direction is indicated by red arrow. There is a slight imaging rotation between the HAADF STEM image and the Lorentz image due to difference in image rotation for the different modes (Color figure online).

the shorter time, i.e., 1.5 minutes, "drops out" of the range with smaller $B_{\mathrm{r}}=0.98 \mathrm{~T}$. These variations may be due to the sample size compared to the grain size. Samples with more grain boundary phases may have lower $B_{\mathrm{r}}$ simply due to a lower phase fraction of the $\alpha_{1}$ phase.

\section{DISCUSSION}

The coarsening of the $\alpha_{1}$ and $\alpha_{2}$ phases and effect of $\mathbf{H}$ during annealing on alloys have been studied extensively. ${ }^{[2,19,20]}$ Theory proposed by Neel ${ }^{[20]}$ and developed by Zijlstra ${ }^{[21,22]}$ believes that application of $\mathbf{H}$ during SD 
will form ellipsoids with axis elongation along field direction at the early stage, then coarsening and elongation will take place. The axes of elongation are

Table I. Calculated $\sin \phi^{*} \cos \theta$ Value of Grains I to III Along Three $\langle\mathbf{1 0 0}\rangle$ Directions

\begin{tabular}{|c|c|c|c|c|c|c|c|c|c|}
\hline & \multicolumn{3}{|r|}{ I } & \multicolumn{3}{|r|}{ II } & \multicolumn{3}{|r|}{ III } \\
\hline & $\phi$ & $\theta$ & $\sin \phi^{*} \cos \theta$ & $\phi$ & $\theta$ & $\sin \phi^{*} \cos \theta$ & $\phi$ & $\theta$ & $\sin \phi^{*} \cos \theta$ \\
\hline$\langle 100\rangle$ & 78 & 2 & 0.977 & 89 & 50 & 0.643 & 56 & 47 & 0.565 \\
\hline$\langle 010\rangle$ & 11 & 29 & 0.167 & 82 & 40 & 0.759 & 54 & 44 & 0.582 \\
\hline$\langle 001\rangle$ & 17 & 61 & 0.142 & 5 & 3 & 0.087 & 37 & 1 & 0.601 \\
\hline
\end{tabular}

parallel to the $\mathbf{H}$ irrespective of its orientation relative to the particular crystallographic direction in the alloy. ${ }^{[22]}$ Another theory developed by Cahn ${ }^{[19]}$ suggested that in the absence of $\mathbf{H}$, the SD consists of three mutually perpendicular waves for cubic system. ${ }^{[15]}$ For SD with $\mathbf{H}$, the shape of the particles is established during the first stage of decomposition, which was determined by the anisotropy of magnetic and elastic energy. If the anisotropy in the magnetic energy is much larger than that in the elastic energy, the geometry of the decomposition will be independent of the crystallographic orientation and the FeCo particles will be elongated along the $\mathbf{H}$ direction. If the anisotropy in the elastic energy is predominant, the particles will be elongated

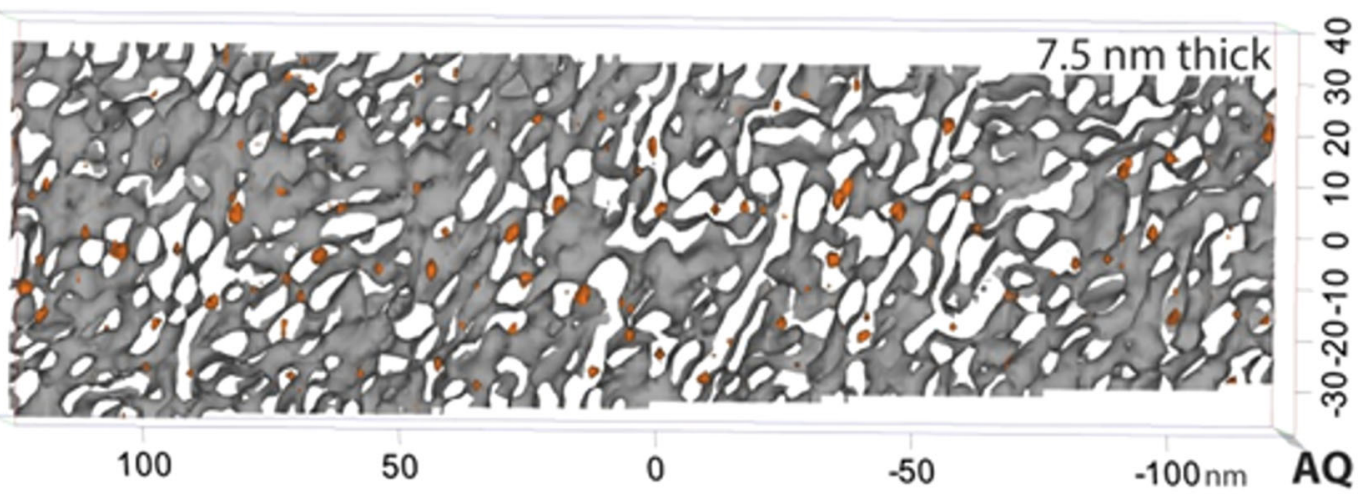

(a)

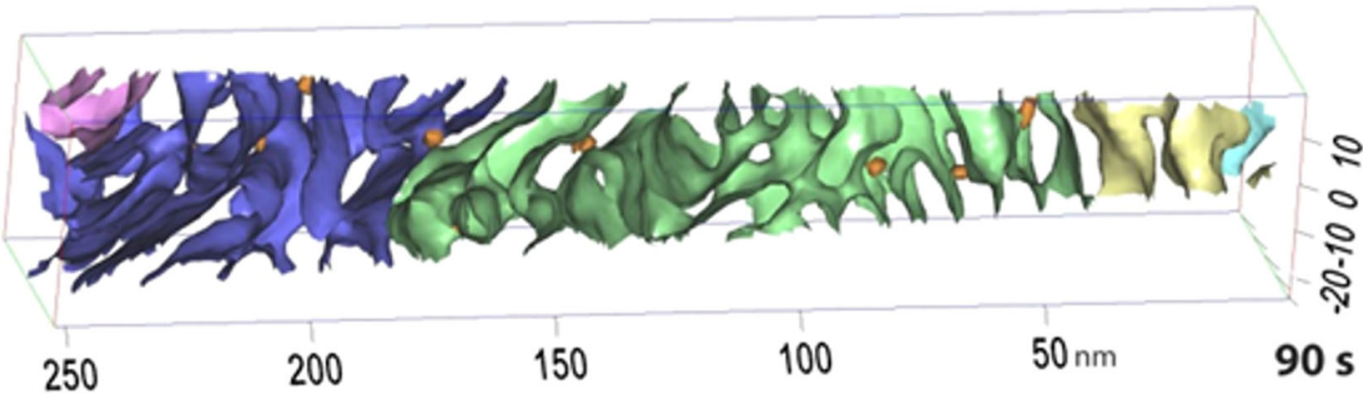

(b)

\section{Each FeCo particle is a different color Cu precipitates $\mathrm{Ni}$ atoms}

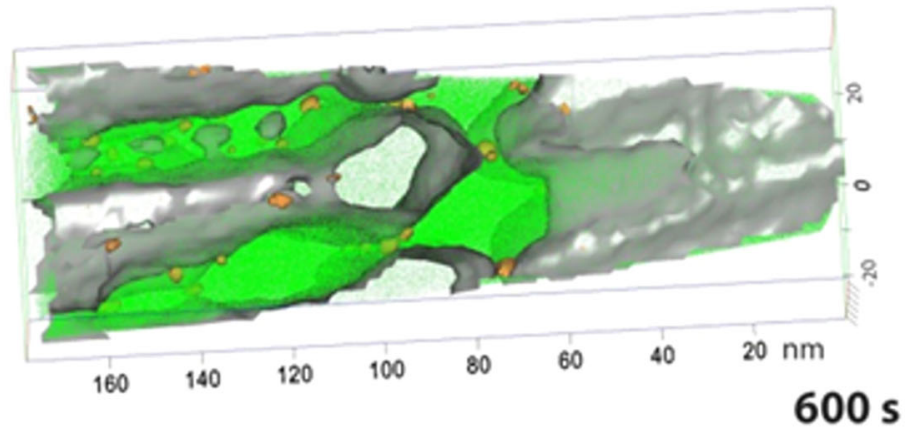

(c)

Fig. 6-30 pct Fe and 7 pct $\mathrm{Cu}$ (orange) concentration isosurfaces for (a) sample A (as-quenched), (b) sample B (90 s), and (c) sample C (600 s aging) depicting the atom probe tomography visualizations of the complex interpenetrating morphology of the FeCo phase and the spheroidal $\mathrm{Cu}$ precipitates, respectively. Due to the fine scale nature of the microstructure of the as-quenched condition, the data in (a) are from a 7.5$\mathrm{nm}$-thick slice extracted from the full volume. The entire FeCo isosurface in the as-quenched condition is a single particle. In (b), a different color has been used for each section of the FeCo concentration isosurfaces within the volume of analysis. Ni atoms have been superimposed in (c) to mark the NiAl phase (Color figure online). 
along the crystallographic direction which minimizes the elastic energy. Cahn also estimated that the elastic energy is much greater than the magnetic energy except near the Curie temperature for alnico. ${ }^{[19]}$ The magnetic shape anisotropy will be further increased only by diffusion of atoms between two phases without changing their shape. ${ }^{[19]}$ This was experimentally observed by Yermolenko et al., who showed that when there was an angle between $\mathbf{H}$ and $\langle 100\rangle$ direction, the axes of $\alpha_{1}$ particles' elongation was not parallel to the field direction as referenced by McCurrie. ${ }^{[2]}$

Our observation of sample A clearly showed that phase morphology forms rapidly but incompletely, resulting in interconnected $\alpha_{1}$ and $\alpha_{2}$ phases, respectively. Since this forms during the rapid radiant/conductive cooling during the quench process, there is no applied magnetic field to bias its growth. The continuity of $\alpha_{1}$ phase acts as a large, single magnetic particle with no discernible shape anisotropy, which accounts for the low coercivity of sample A. Annealing the alloys at $1073 \mathrm{~K}\left(800^{\circ} \mathrm{C}\right)$ with $\mathbf{H}$ will cause coarsening and elongation of the $\alpha_{1}$ phase in any of the three variants of the $\langle 001\rangle$ direction most favorable to the applied field direction. Isolated $\{110\}$ and $\{100\}$ faceted $\alpha_{1}$ phase are

Table II. Measured Chemical Composition in $\alpha_{1}$ and $\alpha_{2}$ Phases for Samples A to $\mathrm{C}$ by Atom Probe

\begin{tabular}{|c|c|c|c|c|c|c|}
\hline \multirow[b]{2}{*}{ Sample } & \multicolumn{3}{|c|}{$\begin{array}{l}\text { FeCo bcc Phase } \\
\text { (at. pct) }\end{array}$} & \multicolumn{3}{|c|}{$\begin{array}{l}\text { AlNi Intermetallic } \\
\text { Phase (at. pct) }\end{array}$} \\
\hline & A & B & $\mathrm{C}$ & A & $\mathrm{B}$ & $\mathrm{C}$ \\
\hline $\mathrm{Fe}$ & 32.75 & 39.7 & 44.98 & 13.15 & 11.65 & 12.74 \\
\hline $\mathrm{Co}$ & 37.17 & 38.36 & 40.42 & 30.23 & 31.5 & 31.48 \\
\hline $\mathrm{Ni}$ & 10.42 & 8.03 & 5.85 & 17.72 & 18.45 & 17.99 \\
\hline $\mathrm{Al}$ & 11.84 & 9.38 & 6.01 & 21.97 & 22.68 & 22.31 \\
\hline $\mathrm{Cu}$ & 2.05 & 1.25 & 1.06 & 3.53 & 2.41 & 2.49 \\
\hline $\mathrm{Ti}$ & 5.53 & 3.11 & 1.45 & 13.05 & 13.04 & 12.66 \\
\hline Volume fraction (pct) & 77.56 & 61.21 & 50.24 & 21.94 & 38.35 & 48.97 \\
\hline \multicolumn{7}{|c|}{$\begin{array}{l}\text { Uncertainty ranges from } 0.002 \text { to } 0.031 \text { at. pct based on estimates } \\
\text { of the uncertainty in the atomic mass computed by time-of-flight } \\
\text { methods, probability of detecting an atom species and total species in } \\
\text { the volume. }\end{array}$} \\
\hline
\end{tabular}

a diffusion-controlled processes and, therefore, improve with increasing annealing time. The short time of exposure to the applied magnetic field demonstrates the rapidity at which this biased growth can occur. More $\mathrm{Cu}$ segregated from the major phases and moved to the corner of two $\alpha_{1}\{110\}$ facets during the coarsening process. Since the edge of two $\{110\}$ facet of $\alpha_{1}$ phase has the largest elastic strain, ${ }^{[15]}$ existence of $\mathrm{Cu}$-rich phase at that point may be able to reduce the total elastic energy of the system by defects formation. The coarsening process should also be thermal dynamically favorable, since the coarsening and isolation of $\alpha_{1}$ phase will not only reduce the magnetostatic energy, but also reduce the interfacial area of the initial single $\alpha_{1}$ particle (reduction of interfacial energy). ${ }^{[2,15]}$ However, there appears to be a balance between the reduction in interfacial energy and total energy of the system which limits coarsening. The optimization of the cuboidal morphology is an interplay between chemistry and processing time and temperature of the drawing process. Decreasing of volume ratio and $\mathrm{Al}, \mathrm{Ni}, \mathrm{Ti}$, and $\mathrm{Cu}$

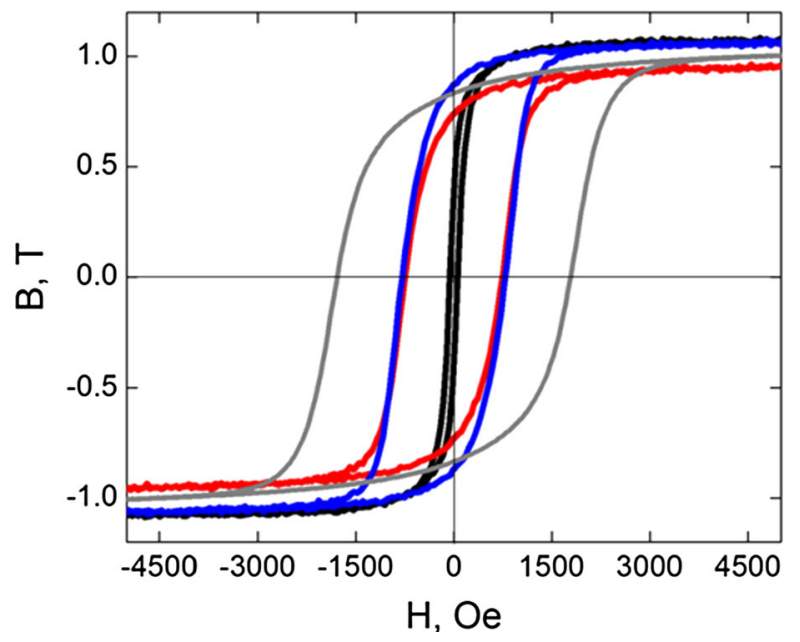

Fig. 8-Magnetic hysteresis of three samples investigated as well as commercial alnico 8: sample A (as-quenched, black), sample B (90 s, red), sample $\mathrm{C}$ (600 s aging, blue), and commercial alnico 8 (gray) (Color figure online).

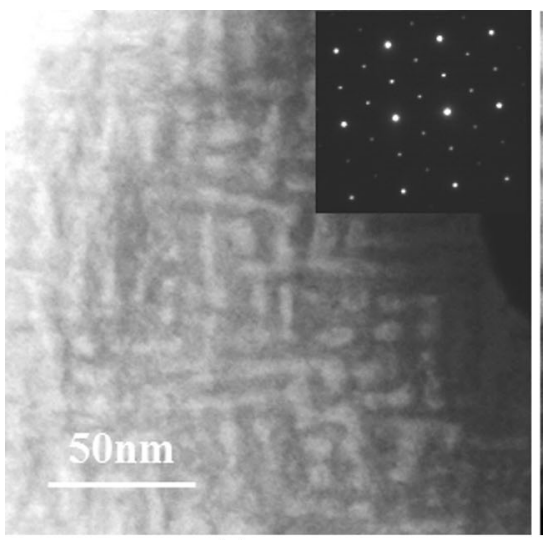

(a)

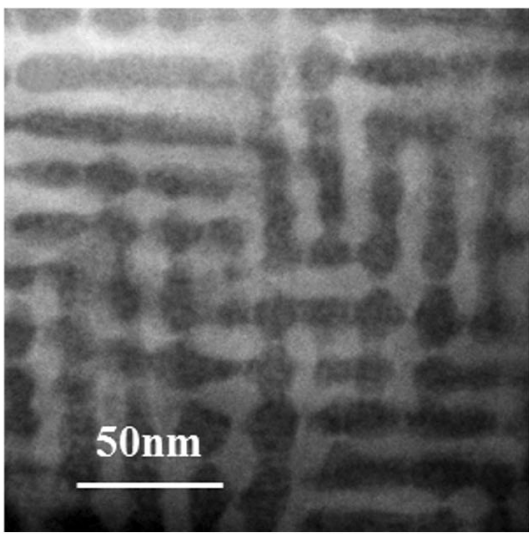

(b)

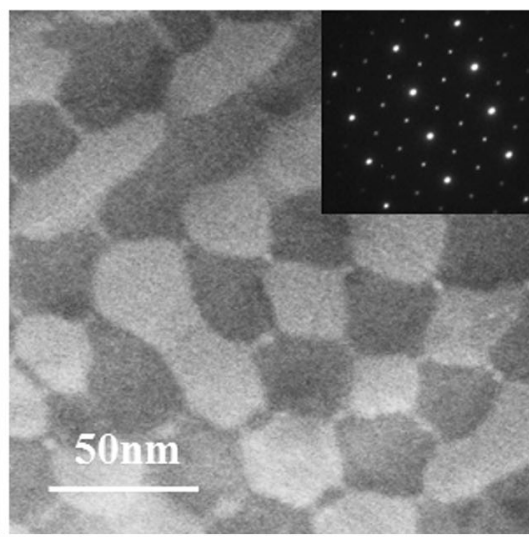

(c)

Fig. 7-HAADF STEM images of Samples A (as-quenched, $a$ ), B (90 s, b), and C (600 s aging, $c$ ). 


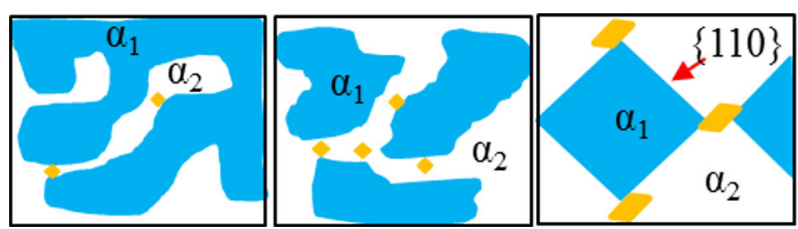

Fig. 9-Schematic of coarsening process during magnetic field annealing in alnico 8 alloy.

concentrations in the $\alpha_{1}$ phase with increasing annealing time may be a result of magnetic shape anisotropy refinement by atom diffusion with external field. ${ }^{[2]}$ The coercivity increase from samples A to B can be explained by the development of the elongated cuboidal $\alpha_{1}$ phase necessary for shape anisotropy. From sample B to C, the coercivity will be further improved by increasing isolation of the $\alpha_{1}$ cuboids and lengthening along the applied field direction as indicated by the increase in $B_{\mathrm{r}}$. However, the diameter of the cuboids are increasing as well, as measured from TEM, which may change the magnetization reversal mechanism of sample $\mathrm{C}$ from coherent rotation to curling when the diameter exceeds $\sim 15 \mathrm{~nm},{ }^{[23]}$ which will reduce the coercivity of the materials. This may explain the similar coercivity for samples $\mathrm{B}$ and $\mathrm{C}$. The isolation of $\alpha_{1}$ phase and segregation of $\mathrm{Cu}$ to the corner of two $\alpha_{1}$ particle is very important for the coercivity improvement of the alloys, due to the reduction of interaction of the $\alpha_{1}$ cuboids during the magnetization process and creation of strain field around those $\mathrm{Cu}$-rich particles. Since size, shape, and isolation of the $\alpha_{1}$ regions are all critical to coercivity, the coarsening of the $\alpha_{1}$ particles which would reduce coercivity may be offset by better isolation so there is a net gain in coercivity with long time anneals. The evolution of $\alpha_{1}$ phase morphology with increasing annealing time projected along [100] zone axis is shown schematically in Figure 9.

The rapid convergence of the nanostructure of the $1073 \mathrm{~K}\left(800^{\circ} \mathrm{C}\right)$ anneal in Figure $7(\mathrm{c})$ to that of the fully processed commercial alloy in Figure 3 shows that the longer processing times used in the commercial alloy heat treatments may involve more subtle refinement of the nanostructuring although the general features are in place after only 10 minutes at $1073 \mathrm{~K}\left(800{ }^{\circ} \mathrm{C}\right)$. The comparison of the compositions of the spinodal phases from APT of the short heat-treated alloys to that of the commercial alloy show some continued diffusion of the major elements between the phases (Table II). Most notable are increase in $\mathrm{Fe}$ and decrease in $\mathrm{Al}$ and $\mathrm{Ti}$ in the $\alpha_{1}$ phase. Well-defined $\mathrm{Cu}$ precipitates at the $\alpha_{1}$ to $\alpha_{2}$ phase boundary are another factor that distinguishes the short and commercially annealed samples. The nearly factor of 2 increase in coercivity is most likely due to these further refinement in both chemistry and surface morphology of the longer anneal. Well-isolated regions of the $\alpha_{1}$ phase with smooth, more regular shaped boundaries would provide the highest coercivity based on the standard shape anisotropy.

Grain alignment also appears to be a factor. Careful examination of the commercial alnico 8 alloy along longitudinal direction showed that the $\alpha_{1}$ phase is elongated along all three $\langle 100\rangle$ directions with projected $\mathbf{H}(\cos (\theta) \sin (\phi) \neq 0)$. The $\langle 100\rangle$ crystallographic direction with the largest projected $\mathbf{H}$ will grow dominantly and longest. The overall orientation of $\alpha_{1}$ cuboids in alnico 8 is random with a length ranging from tens of nanometers to microns. If these cuboids are too close or worse yet, connect to their neighbors, these regions would result in easy cascade of reversal domains in a demagnetizing field. It appears that all the factors of the nanostructuring are required; size shape and spacing as well as orientation of the crystallographic axis to the applied field. Long anneals are necessary to achieve the ideal interfaces.

\section{CONCLUSIONS}

Microstructural study of commercial and heat-treated alnico 8 alloys showed that the phase evolution proceeds rapidly. Initial quench from $1523 \mathrm{~K}\left(1250{ }^{\circ} \mathrm{C}\right)$ forms an irregular but continuous network structure. A 90 seconds anneal at $1073 \mathrm{~K}\left(800^{\circ} \mathrm{C}\right)$ with a $1 \mathrm{~T}$ magnetic field annealing is sufficient to begin forming coarser, faceted, and increasing isolated cuboids of the $\alpha_{1}$ phase necessary for coercivity. Long time anneals are required to achieve optimal coercivity. Whereas $\mathrm{Cu}$ is unstable in either phase, with time $\mathrm{Cu}$ segregates and diffuses to the corner of two $\{110\}$ facets of the $\alpha_{1}$ phase during the heattreatment process. Application of $\mathbf{H}$ biased the elongation of $\alpha_{1}$ rod along the $\langle 100\rangle$ direction with the largest projected $\mathbf{H}$ field. The final spinodal phase distribution and micromagnetic domain structure is closely related to the relative orientation relationship between $\mathbf{H}$ and grain orientation. Having a complete picture of how the nanostructuring proceeds during the anneal and a clear idea of how the orientation of the applied magnetic field to the crystallographic orientation can alter the spinodal provide testable ideas for both experimental and theoretical optimization of this alloy. Tilting of the applied field to the crystallographic alters the uniformity of the spinodal which in turn alters coercivity.

\section{ACKNOWLEDGMENTS}

Research in Ames Lab was supported by U.S. DOE, Office of Energy Efficiency and Renewable Energy (EERE), under its Vehicle Technologies Program, through the Ames Laboratory, Iowa State University under contract DE-AC02-07CH11358. Atom probe tomography research (MKM) was supported through a user project supported by ORNL's Center for Nanophase Materials Sciences (CNMS), which is sponsored by the Scientific User Facilities Division, Office of Basic Energy Sciences, U.S. Department of Energy.

\section{REFERENCES}

1. M.J. Kramer, R.W. McCallum, I.A. Anderson, and S. Constantinides: JOM, 2012, vol. 64 (7), pp. 752-763.

2. E.P. Wohlfarth: Ferromanetic Materials, vol. 3, chap. 3, NorthHolland Publishing Company, 1982. 
3. M. Takeuchi and Y. Iwama: Trans. Jpn Inst. Met., 1976, vol. 17 (8), pp. 489-496.

4. Y. Iwama and M. Takeuchi: Trans. Jpn Inst. Met., 1974, vol. 15 (5), pp. 371-377.

5. M.G. Hetherington, A. Cerezo, J.P. Jakubovics, and G.D.W. Smith: J. Phys. Paris, 1988, vol. 49 (C-8), pp. 673-674.

6. J.J. Mason, D.W. Ashall, and A.V. Dean: IEEE Trans. Magn. Magn., 1970, vol. 6 (2), pp. 191-194.

7. Y.B. Kim, S.A. Song, and T.K. Kim: IEEE Trans. Magn., 1987, vol. 23 (4), pp. 1952-1955.

8. M.G. Hetherington, A. Cerezo, J.P. Jakubovics, and G.D.W. Smith: J. Phys. Paris, 1984, vol. 45 (Nc9), pp. 429-434.

9. S.A. Cowley, M.G. Hetherington, J.P. Jakubovics, and G.D.W. Smith: J. Phys. Paris, 1986, vol. 47 (C-7), pp. 211-216.

10. J.M. Aquino, C.D. Rios, M.M. Yoshida, and O.A. Valenzuela: Magn. Magn. Mater. Appl., 1999, vols. 302-303, pp. 329-333.

11. M.K. Miller and R.G. Forbes: Mater. Charact., 2009, vol. 60 (6), pp. $461-469$.

12. D.W. Saxey, J.M. Cairney, D. McGrouther, T. Honma, and S.P. Ringer: Ultramicroscopy, 2007, vol. 107 (9), pp. 756-760.
13. Q. Xing, M.K. Miller, L. Zhou, H.M. Dillon, R.W. McCallum, I.E. Anderson, S. Constantinides, and M.J. Kramer: IEEE Trans. Magn., 2013, vol. 49 (7), pp. 3314-3317.

14. D.B. Williams and C.B. Carter: Transmission Electron Microscopy, Plenum Press, New York, 1996.

15. J.W. Cahn: Acta Metall. Mater., 1962, vol. 10, pp. 179-183.

16. J.W. Cahn and J.E. Hilliard: Acta Metall. Mater., 1971, vol. 19 (2), pp. 151-161.

17. X.Y. Sun, C.L. Chen, L. Yang, L.X. Lv, S. Atroshenko, W.Z. Shao, X.D. Sun, and L. Zhen: J. Magn. Magn. Mater., 2013, vol. 348 , pp. $27-32$

18. O.C. Hellman, J.A. Vandenbroucke, J. Rusing, D. Isheim, and D.N. Seidman: Microsc. Microanal., 2000, vol. 6 (5), pp. 437444.

19. J.W. Cahn: J. Appl. Phys., 1963, vol. 34 (12), pp. 3581-3586.

20. L. Neel: Cr. Hebd. Acad. Sci., 1947, vol. 225 (2), pp. 109-111.

21. H. Zijlstra: J. Appl. Phys., 1956, vol. 27 (10), pp. 1249-1250.

22. H. Zijlstra: J. Appl. Phys., 1961, vol. 32 (3), pp. 194S-196S.

23. H. Zeng, R. Skomski, L. Menon, Y. Liu, S. Bandyopadhyay, and D.J. Sellmyer: Phys. Rev. B, 2002, vol. 65 (13), p. 134426. 